\title{
Atividade física e indicadores de saúde em pessoas vivendo com HIV/aids
}

\section{Physical activity and health indicators in people living with HIV/aids}

\author{
Hygor Cordeiro ${ }^{1}$ Lidyane Midori Kitagawa¹, Milena Arantes Máximo, Douglas Fernando Dias², Débora Alves Guariglia1,3,4 \\ ${ }^{1}$ Faculdade Estácio de Sá de Ourinhos (FAESO), Ourinhos (SP), Brasil. \\ ${ }^{2}$ Programa de Pós-Graduação em Saúde Coletiva, Universidade Estadual de Londrina (UEL), Londrina (PR), Brasil. \\ ${ }^{3}$ Programa de Pós-Graduação em Educação Física, Universidade Estadual de Maringá (UEM), Maringá (PR), Brasil. \\ ${ }^{4}$ Universidade Estadual do Norte do Paraná (UENP), Jacarezinho (PR), Brasil.
}

DOI: http://dx.doi.org/10.7322/abcshs.v43i3.1000

\section{RESUMO}

Introdução: O estilo de vida ativo pode contribuir de forma expressiva em pessoas vivendo com HIV/aids auxiliando na melhoria das capacidades físicas e combate aos efeitos adversos da medicação. Objetivo: Analisar a associação entre atividade física e fatores sociodemográficos e de saúde em pessoas vivendo com HIV/ aids do município de Ourinhos-SP. Métodos: Foram entrevistados pacientes em tratamento no Serviço de Atendimento Especializado em Doenças Infecciosas (SAEDI). A atividade física habitual e seus domínios foram avaliados mediante o questionário de Baecke, além disso, os prontuários dos pacientes foram acessados para coleta de dados adicionais de saúde. Resultados: Como principais resultados, observou-se baixos níveis de atividade física de lazer na população investigada (21,0\%). Modelos de regressão de Poisson ajustados por fatores de confundimento identificaram associação entre atividade física ocupacional e maior classe social (RP: 3,32; IC95\%: 1,34-8,25); atividades físicas de lazer e locomoção e maior pressão arterial diastólica (PAD) (RP: 3,17; IC95\%: 1,43-7,07); atividade física habitual e tempo de diagnóstico superior a 150 meses (RP: 3,18; IC95\%: 1,16-8,72) e maior prevalência de exercícios físicos no lazer associou-se a maior classe social (RP: 2,86; IC95\%: 1,11-7,36). Conclusão: Pessoas vivendo com HIV/aids em tratamento na região de Ourinhos apresentam baixos níveis de atividade física. Além disso, classe social, tempo de diagnóstico e PAD apresentaram relações com atividade física

Palavras-chave: Síndrome de imunodeficiência adquirida; Atividade motora; prioridades em saúde.

\begin{abstract}
Introduction: An active lifestyle may contribute significantly to people living with HIV/aids by improving physical abilities and reducing the adverse effects of medications. Objective: To analyze the association between physical activity and sociodemographic and health factors in people living with HIV/ aids in the municipality of Ourinhos-SP. Methods: Patients undergoing treatment were interviewed at the Specialized Infectious Diseases Care Service. Habitual physical activity and its domains were evaluated using the Baecke questionnaire and patients' charts were consulted for additional health data. Results: The proportion of individuals undertaking leisure physical activity in our sample was low (21\%). Poisson regression models, adjusted for confounding factors, identified associations between occupational physical activity and higher social class (PR: 3.32; 95\% Cl: 1.34-8.25); between leisure physical activity and locomotion and higher diastolic blood pressure (DBP) (PR: 3.17; 95\% Cl: 1.43-7.07); and between habitual physical activity and duration of diagnosis >150 months (PR: 3.18; 95\% Cl: 1.16-8.72) and higher prevalence of leisure physical activities was associated with higher social class (PR: $2.86,95 \% \mathrm{Cl}: 1.11$ 7.36). Conclusion: People living with HIV/aids and undergoing treatment in the Ourinhos region have low levels of physical activity. Furthermore, social class, duration of diagnosis and DBP were found to be associated with physical activity.
\end{abstract}

Keywords: Acquired Immunodeficiency Syndrome; motor activity; health priorities.

Recebido em: 23/07/2017

Revisado em: 10/01/2018

Aprovado em: 04/05/2018

Autor para correspondência: Débora Alves Guariglia - Faculdade Estácio de Sá de Ourinhos - Avenida Luís Saldanha Rodrigues, s/n, Nova Ourinhos CEP: 19907-510 - Ourinhos (SP), Brasil - E-mail: debora.guariglia@gmail.com

Conflito de interesses: nada a declarar 


\section{INTRODUÇÃO}

O HIV é um vírus que ao infectar o corpo humano é capaz de destruir os linfócitos TCD4, deixando o corpo vulnerável a infecções e doenças oportunistas. A síndrome da imuno deficiência adquirida (aids) é o estágio mais avançado da infecção pelo HIV. Dessa forma, uma pessoa contaminada pelo vírus tem o diagnóstico da aids quando passa a ter uma contagem de linfócitos ${ }^{1}$ TCD4 menor que 200 células $/ \mathrm{mm}^{3}$.

De 1980 ao final de 2016, foram notificados no país 842.710 casos de aids. Só no Brasil são registrados anualmente uma média de 41,1 mil casos nos últimos cinco anos ${ }^{2}$.

Com o avanço do tratamento e a utilização de combinação de medicamentos da terapia antirretroviral (TARV) as pessoas vivendo com $\mathrm{HIV} /$ aids passaram a ter uma maior expectativa de vida, porém, muitas vezes. Convivem com alguns efeitos adversos derivados do tratamento medicamentoso. Os principais efeitos adversos da HAART são a síndrome metabólica e a lipodistrofia. Adicionalmente, também é comum observar efeitos psicológicos como agitação, confusão, ansiedade, pesadelos, alucinações e depressão, somadas aos efeitos físicos e metabólicos já citados, acarretam a uma menor qualidade de vida a essa população ${ }^{3}$. Dessa forma, muitas pessoas em vez de recorrer a mais medicamentos para contornar os sintomas, buscam auxílio em terapias alternativas como a prática de exercícios físicos ${ }^{4}$.

A recomendação de uma vida ativa se aplica a população em geral independente da disfunção apresentada. Para pessoas vivendo com HIV não é diferente, atividades físicas regulares têm se mostrado benéficas para esta população $0^{5-7}$. Um estudo de coorte demonstrou que pessoas recém-diagnosticadas com HIV que fazem atividade física de forma sistematizada apresentam melhor aceitação da doença e tem a progressão do diagnóstico da aids mais tardia em relação a mesma população sedentária ${ }^{5}$.

Além disso, estudos recentes demonstram que pessoas vivendo com HIV com uma melhor capacidade aeróbica estão menos propensos a transtornos neurocognitivos ${ }^{6}$. Atividades físicas sistematizadas, como exercícios aeróbicos ou treinamento concorrente (aeróbico e resistido) três vezes por semana proporcionam benefícios como melhora da capacidade cardiorrespiratória, composição corporal, força e na qualidade de vida desta população, além de não apresentarem nenhum maleficio ${ }^{7}$.

É importante ressaltar que se manter ativo não se refere diretamente a realização de exercícios físicos no lazer (atividade física sistematizada). Essa tarefa pode ser feita aumentando os níveis de atividade física em sua ocupação (no trabalho, nos estudos), ou ainda na locomoção (adotando sua locomoção de forma ativa, se deslocando ao trabalho, ao lazer, as compras a pé ou de bicicleta, entre outros).

Portanto, identificar os principais fatores associados à prática de atividade física total e de seus diferentes domínios (ocupacional, lazer, transporte, etc.) em pessoas vivendo com HIV/aids poderá favorecer tanto o aprofundamento do conhecimento sobre essa temática quanto subsidiar a criação de estratégias para o cuidado dessa população, mediante a promoção de um estilo de vida mais ativo. Sendo assim, o objetivo do presente estudo foi analisar a associação entre atividade física e fatores sociodemográficos e de saúde em pessoas vivendo com HIV/aids em tratamento no município de Ourinhos-SP.

\section{MÉTODOS}

Trata-se de um estudo epidemiológico observacional do tipo transversal desenvolvido no serviço de atendimento especializado em doenças infecciosas (SAEDI) na cidade de Ourinhos-SP. Como critérios de inclusão os sujeitos deveriam: ser diagnosticados com HIV ou aids, estar em tratamento no SAEDI, concordar em responder os questionários, ter mais de 18 anos e assinar o termo de consentimento livre e esclarecido. Além disso, foram excluídas as pessoas que não preencheram corretamente o questionário ou que não conseguiram responder todos os questionários no dia da coleta de dados. O presente estudo foi aprovado pelo comitê de ética do hospital 9 de julho (CAAE: 449912115.3.0000.5455) - São Paulo.

No período da coleta o SAEDI registrava 335 pacientes em tratamento com HIV/aids, sendo 158 do sexo masculino e 177 do feminino. Todas as pessoas que preencheram os critérios de inclusão e foram atendidas de outubro de 2015 a fevereiro de 2016 foram consideradas elegíveis e convidadas para o estudo. No entanto, aproximadamente $10 \%$ dos indivíduos abordados não tiveram interesse em participar da pesquisa. Assim, a amostra do presente estudo foi composta de 71 voluntários, que representa $21 \%$ dos pacientes atendidos atualmente no município de Ourinhos-SP.

Foi utilizado o questionário de atividade física habitual ${ }^{8} \mathrm{o}$ qual foi validado no Brasil para pessoas vivendo com HIV/aids?. O questionário é composto de 16 questões que abrangem três escores de atividade física habitual (AFH) dos últimos 12 meses: 1) escore de atividades físicas ocupacionais (AFO) com oito questões, 2) escore de exercícios físicos no lazer (EFL) com quatro questões, 3) escore de atividades físicas de lazer e locomoção (ALL) com quatro questões. Adicionalmente, por meio das informações respondidas nas questões sobre EFL foram estimadas quais pessoas atendiam ou não os níveis recomendados de atividade física sistematizada no lazer $\left(\geq 150 \mathrm{~min} / \mathrm{sem}\right.$ de EFL) ${ }^{10}$. Também foram coletadas indicadores de informações socioeconômicas da amostra por meio do questionário validado da $\mathrm{ABEP}^{11}$.

Em uma segunda etapa da pesquisa os prontuários dos pacientes foram acessados e algumas informações pertinentes foram coletadas, como: peso da última consulta; dados sobre a doença; data de diagnóstico, data de início do tratamento, tipo de medicação utilizado atualmente, doenças oportunistas, dados sobre as aferições da pressão arterial nas últimas consultas; e dados sobre carga viral, TCD4 e TCD8 nos últimos exames. Para todas essas variáveis coletadas do prontuário foram testadas as relações com os domínios de atividade física. 
Os dados descritivos foram apresentados em frequência absoluta e relativa ou em média e desvio padrão. Para as análises bivariadas foi utilizado o teste do qui-quadrado, ao passo que para as análises multivariáveis entre atividade física (AFO, EFL, ALL e AFH), variáveis sociodemográficas e de saúde, foi empregada a regressão de Poisson com ajuste robusto de variância. Foram consideradas elegíveis para o modelo multivariável apenas os fatores que apresentaram $P<20,0$ nas análises bivariadas. Tal modelo foi ajustado pelas seguintes variáveis sociodemográficas: sexo, idade e classe social. A seleção das variáveis de ajuste seguiu critério teórico, ou seja, a existência de evidência na literatura de que poderiam confundir as associações de interesse por estarem relacionadas tanto com a atividade física quanto com as variáveis independentes investigadas. Para todas as análises (bivariadas e multivariáveis) a significância adotada foi de $P \leq 0,05$ e o programa utilizado foi o SPSS 20.0 .

\section{RESULTADOS}

Dentre os resultados apresentados na tabela 1 observa-se que a categoria que apresentou a maior frequência foi do sexo feminino, casados, com idade entre 51 e 60 anos, da classe socioeconômica C, possuindo IMC $>25 \mathrm{~kg} / \mathrm{m}^{2}$, com ensino fundamental I, abaixo dos níveis de EFL, fazem uso de medicamentos inibidores de protease, com carga viral detectável nos exames. Além disso, os sujeitos apresentaram a contagem de linfócitos TCD4 e TCD8 em média de 663 e 1012,9 cópias respectivamente, pressão arterial sistólica de 118,7 mmHg e diastólica de $80,2 \mathrm{mmHg}$ e um tempo médio de diagnóstico de 150,5 meses (Tabela 1).

A tabela 2 demonstra a associação entre atividade física (AFO, EFL, ALL e AFH) e variáveis sociodemográficas de saúde. As maiores prevalências de AFO e ALL estiveram associadas, respectivamente, a maior classe social e PAD maior que $80 \mathrm{mmHg}$. Para as outras variáveis não foi identificada associação estatisticamente significativas.

Após ajuste para variáveis de confundimento, tanto AFO quanto ALL mantiveram-se associadas, respectivamente, a maior classe social (RP ajustada: 3,32; IC95\%: 1,34-8,25) e PAD maior que $80 \mathrm{mmHg}$ (RP ajustada: 3,17; IC95\%: 1,43-7,07) (Tabela 3). Além disso, a maior prevalência de EFL associou-se a maior classe social (RP ajustada: 2,86; IC95\%: 1,11-7,36). Também foi verificado que a prevalência de AFH foi 3,18 vezes superior entre os que tinham tempo de diagnóstico superior a 150 meses em relação aqueles com tempo inferior a 50 meses (Tabela 3 ).

\section{DISCUSSÃO}

O objetivo do presente estudo foi analisar a associação entre atividade física e fatores sociodemográficos e de saúde em pessoas vivendo com HIV/aids em tratamento no município de OurinhosSP. Como principais resultados observou-se que apenas $21 \%$ da população investigada atendem as recomendações de atividade física sistematizada no lazer preconizados pelo $\mathrm{ACSM}^{10}$. Além disso, verificou-se que (independente de fatores de confundimento) as maiores prevalências de AFO, ALL e AFH associaram-se,

Tabela 1: Características descritivas da amostra de pessoas vivendo com HIV/aids no município de Ourinhos, SP, 2015-2016 ( $n=71)$.

\begin{tabular}{|c|c|c|}
\hline Variáveis & \multicolumn{2}{|c|}{$\%$} \\
\hline \multicolumn{3}{|l|}{ Sexo } \\
\hline Feminino & \multicolumn{2}{|c|}{52,1} \\
\hline Masculino & \multicolumn{2}{|c|}{47,9} \\
\hline \multicolumn{3}{|l|}{ Faixa etária (anos) } \\
\hline $20-30$ & \multicolumn{2}{|c|}{8,4} \\
\hline $31-40$ & \multicolumn{2}{|c|}{16,9} \\
\hline $41-50$ & \multicolumn{2}{|c|}{29,6} \\
\hline $51-60$ & \multicolumn{2}{|c|}{35,2} \\
\hline $61-70$ & \multicolumn{2}{|c|}{9,9} \\
\hline \multicolumn{3}{|l|}{ Classe social } \\
\hline$A$ & \multicolumn{2}{|c|}{4,2} \\
\hline B & \multicolumn{2}{|c|}{35,2} \\
\hline C & \multicolumn{2}{|c|}{50,7} \\
\hline $\mathrm{D} / \mathrm{E}$ & \multicolumn{2}{|c|}{9,9} \\
\hline \multicolumn{3}{|l|}{ IMC $\left(\mathrm{kg} / \mathrm{m}^{2}\right)$} \\
\hline$<25$ & \multicolumn{2}{|c|}{47,9} \\
\hline$>25$ & \multicolumn{2}{|c|}{52,1} \\
\hline \multicolumn{3}{|l|}{ Escolaridade } \\
\hline Analfabeto & \multicolumn{2}{|c|}{14,0} \\
\hline Ensino fundamental 1 & \multicolumn{2}{|c|}{33,8} \\
\hline Ensino fundamental 2 & \multicolumn{2}{|c|}{15,5} \\
\hline Ensino médio & \multicolumn{2}{|c|}{25,4} \\
\hline Superior & & \\
\hline Estado Civil & & \\
\hline Solteiro & & \\
\hline Amasiado & & \\
\hline Casado & & \\
\hline Divorciado & & \\
\hline Viúvo & & \\
\hline Não informado & & \\
\hline EFL ( $\geq 150 \mathrm{~min} / \mathrm{sem})$ & & \\
\hline Sim & & \\
\hline Não & & \\
\hline Medicação & & \\
\hline NNRTI & & \\
\hline IP & & \\
\hline II & & \\
\hline Carga viral & & \\
\hline Detectado & & \\
\hline Indetectável & & \\
\hline Variáveis & Média & DP \\
\hline Contagem de linfócitos: & & \\
\hline TCD4 Cópias & 663 & 341 \\
\hline TCD8 Cópias & 1012,9 & 484,4 \\
\hline Pressão arterial (mmhg) & & \\
\hline sistólica & 118,7 & 14,4 \\
\hline diastólica & 80,2 & 10,2 \\
\hline Tempo de diagnóstico (meses) & 150,5 & 235,7 \\
\hline
\end{tabular}

Legenda: IMC: índice de massa corporal. EFL: Exercício físico no Lazer. NNRTI: Inibidores Nucleosídeos da Transcriptase Reversa. IP: Inibidores de protease. II: Inibidor de integrasse. DP: Desvio padrão. 
respectivamente, à maior classe social, $\mathrm{PAD}>80 \mathrm{mmHg}$ e tempo de diagnóstico superior a 150 meses. Também foi constatado que o grupo de pessoas com melhor saúde percebida e maior classe social apresentou maior prevalência de EFL.

É evidente na literatura a importância da prática de atividade física e dos benefícios de se manter ativo fisicamente para o bem-estar físico e psicológico para essa população ${ }^{3-5,7}$. No entanto, em virtude da descoberta da sorologia, pessoas vivendo com $\mathrm{HIV} /$ aids podem restringir seu círculo social, atividades no trabalho, lazer e locomoção, em função, sobretudo, do preconceito por se tratar de um agravo à saúde ligado a estigmas e estereótipos ${ }^{12}$. Tal cenário parece contribuir para uma baixa adesão a prática de atividade física ${ }^{13}$ Essa hipótese foi parcialmente confirmada no presente estudo, haja vista que apenas $21 \%$ dos sujeitos investigados atendiam as recomendações de EFL, resultados semelhantes a outros estudos com essa população ${ }^{13}$.

Independente de fatores de confundimento a prevalência de ALL foi 3,17 vezes superior entre aqueles com $\mathrm{PAD}>80 \mathrm{mmHg}$. Esse resultado vai de encontro com a literatura que vem demonstrando a importância da prática de atividade física para o controle

Tabela 2: Associação entre atividade física e variáveis sociodemográficas e de saúde em amostra de pessoas vivendo com HIV/aids no município de Ourinhos, SP, 2015-2016 ( $n=71)$.

\begin{tabular}{|c|c|c|c|c|}
\hline Variável & $\begin{array}{c}\text { AFO } \\
\text { n (\%) }\end{array}$ & $\begin{array}{c}\text { EFL } \\
\text { n (\%) }\end{array}$ & $\begin{array}{c}\text { ALL } \\
\text { n (\%) }\end{array}$ & $\begin{array}{c}\text { AFH } \\
\text { n (\%) }\end{array}$ \\
\hline Total & $16(22,5)$ & $13(18,3)$ & $16(22,5)$ & $23(32,4)$ \\
\hline \multicolumn{5}{|l|}{ Sexo } \\
\hline Masculino & $7(20,6)$ & $9(26,5)$ & $7(20,6)$ & $11(32,4)$ \\
\hline Feminino & $9(24,3)$ & $4(10,8)$ & $9(24,3)$ & $12(32,4)$ \\
\hline $\mathrm{P}$ & 0,707 & $0,126^{a}$ & 0,707 & 0,994 \\
\hline \multicolumn{5}{|l|}{ Idade (anos) } \\
\hline$<50$ & $10(25,6)$ & $6(15,4)$ & $9(23,1)$ & $13(33,3)$ \\
\hline$>50$ & $6(18,8)$ & $7(21,9)$ & $7(21,9)$ & $10(31,2)$ \\
\hline $\mathrm{P}$ & 0,489 & 0,482 & 0,904 & 0,852 \\
\hline \multicolumn{5}{|l|}{ Formação } \\
\hline Analfabeto/ EF1 & $5(14,7)$ & $5(14,7)$ & $9(26,5)$ & $10(29,4)$ \\
\hline EF2 / Médio / Superior & $11(29,7)$ & $8(21,6)$ & $7(18,9)$ & $13(35,1)$ \\
\hline$P$ & 0,130 & 0,452 & 0,447 & 0,607 \\
\hline \multicolumn{5}{|l|}{ Classe Social } \\
\hline$A$ e $B$ & $11(39,3)$ & $8(28,6)$ & $6(21,4)$ & $11(39,3)$ \\
\hline$C, D$ e $E$ & $5(11,6)$ & $5(11,6)$ & $10(23,3)$ & $12(27,9)$ \\
\hline$P$ & $0,006^{*}$ & 0,071 & 0,857 & 0,317 \\
\hline \multicolumn{5}{|l|}{ Tempo de tratamento } \\
\hline $1^{\circ}$ tercil (<35 meses) & $5(20,8)$ & $4(16,7)$ & $2(8,3)$ & $5(20,8)$ \\
\hline $2^{\circ}$ e $3^{\circ}$ tercil ( $\geq 35$ meses) & $11(23,4)$ & $9(19,1)$ & $14(29,8)$ & $18(38,3)$ \\
\hline $\mathrm{P}$ & 0,806 & $1,000^{\mathrm{a}}$ & $0,069^{a}$ & 0,137 \\
\hline \multicolumn{5}{|l|}{ Tempo de diagnóstico } \\
\hline $1^{\circ}$ tercil ( $<50$ meses) & $5(20,8)$ & $5(20,8)$ & $2(8,3)$ & $4(16,7)$ \\
\hline $2^{\circ}$ e $3^{\circ}$ tercil ( $\geq 50$ meses) & $11(23,4)$ & $8(17,0)$ & $14(29,8)$ & $19(40,4)$ \\
\hline $\mathrm{P}$ & 0,806 & 0,694 & $0,069^{a}$ & $0,061^{a}$ \\
\hline \multicolumn{5}{|c|}{ Pressão arterial sistólica $(\mathrm{mm} / \mathrm{Hg})$} \\
\hline$\leq 120$ & $6(31,6)$ & $2(10,5)$ & $5(26,3)$ & $17(32,7)$ \\
\hline$>120$ & $10(19,2)$ & $11(21,2)$ & $11(21,2)$ & $6(31,6)$ \\
\hline$P$ & 0,270 & $0,491^{a}$ & 0,645 & 0,929 \\
\hline \multicolumn{5}{|c|}{ Pressão arterial diastólica $(\mathrm{mm} / \mathrm{Hg})$} \\
\hline$\leq 80$ & $11(19,3)$ & $11(19,3)$ & $9(15,8)$ & $16(28,1)$ \\
\hline$>80$ & $5(35,7)$ & $2(14,3)$ & $7(50,0)$ & $7(50,0)$ \\
\hline $\mathrm{P}$ & 0,188 & $1,000^{\mathrm{a}}$ & $0,006^{*}$ & 0,116 \\
\hline \multicolumn{5}{|l|}{ TCD4 } \\
\hline$<300$ & $2(14,3)$ & $4(28,6)$ & $2(14,3)$ & $3(21,4)$ \\
\hline$\geq 300$ & $13(23,2)$ & $9(16,1)$ & $14(25,0)$ & $20(35,7)$ \\
\hline$P$ & $0,718^{a}$ & $0,277^{a}$ & $0,393^{a}$ & $0,361^{a}$ \\
\hline \multicolumn{5}{|l|}{ Carga viral } \\
\hline Não detectada & $14(27,5)$ & $10(19,6)$ & $12(23,5)$ & $17(33,3)$ \\
\hline Detectada & $2(10,0)$ & $3(15,0)$ & $4(20,0)$ & $6(30,0)$ \\
\hline $\mathrm{P}$ & 0,205 & $0,746^{a}$ & $1,000^{a}$ & 0,787 \\
\hline
\end{tabular}

Legenda: AFO: Atividade física ocupacional; EFL: exercício físico no lazer; ALL: atividade física de lazer e locomoção; AFH: atividade física habitual; PAS: Pressão arterial sistólica. PAD: Pressão arterial diastólica; ${ }^{*} P<0,05$; ${ }^{\circ}$ Correção de Yates. 
da pressão arterial ${ }^{14}$ Por se tratar de um estudo do tipo transversal, em que a temporalidade dos eventos não é conhecida (exposições e desfechos coletamos no mesmo momento), é possível que esse achado possa ser explicado pela causalidade reversa. Ou seja, é plausível supor que pessoas que apresentam elevada PAD tenham iniciado a prática de atividade física no lazer ou no deslocamento com o objetivo de controlar a pressão arterial elevada.

Sujeitos com maior tempo de diagnostico apresentaram prevalência de AFH 3,18 vezes superior em relação aqueles com menor tempo. Esse achado pode se dar pela aceitação da doença onde a pessoa deixa de ter vergonha e começa a ter esperança e apoio da família o que se mostra como um fator significativo para a melhoria da qualidade de vida nessa população ${ }^{15}$. Com passar do tempo os pacientes podem perceber que a doença pode ser tratada e a vida ter continuidade ${ }^{12}$.

Os indivíduos das classes A e B, inesperadamente, se mostraram mais ativos em suas ocupações do que aqueles das classes D e E. Uma possível explicação para esse resultado é que as classes D e E podem ter sido compostas predominantemente por aposentados no presente estudo, o que diminui acentuadamente os níveis de atividade física ocupacional, fator esse que explica o desacordo com a literatura que consistentemente aponta maior prevalência de AFO entre trabalhadores com menor nível socioeconômico ${ }^{16}$.

A prevalência de EFL foi 2,86 superior entre aqueles de maior classe social e 3,43 vezes maior entre aqueles com melhor condição de saúde. Ambos os resultados corroboram a literatura ${ }^{17} \mathrm{e}$ chama a atenção para a relevância do investimento e ampliação de políticas públicas de redução de iniquidades em saúde e promoção de justiça social no Brasil, especialmente em momentos de maior vulnerabilidade social acarretados por crise econômica, tal como o que o país passa neste momento.

Todos esses resultados apontam para importância da criação de estratégias de atendimento multidisciplinar a essa população, não restringindo o tratamento do paciente somente a médicos e enfermeiros. Nessa perspectiva, entende-se que as equipes multiprofissionais - incluindo os profissionais de educação física presentes nas Unidades Básicas de Saúde podem e devem estar preparados atender essa população que pode, eventualmente, necessitar de estratégias diferenciadas.

No entanto, algumas considerações metodológicas são importantes para a interpretação dos resultados e discussão. O presente estudo trata-se de um delineamento transversal, o qual mostra relações, e não uma relação de causa e efeito. Delineamentos longitudinais podem proporcionar essas respostas. Além disso, foram voluntários pouco mais de $20 \%$ dos pacientes atendidos pelo SAEDI de Ourinhos e sabe-se que algumas associações ficaram prejudicadas devido ao baixo número de sujeitos, no entanto muitos dos pacientes preferiram não participar para preservar sua identidade. Estudos com esta população não são comuns na cidade de Ourinhos, encontrando assim alguma resistência por parte da população por não estar habituada com este tipo de estudo. Além disso, deve-se se considerar que a amostra foi intencional o que pode sugere uma estimativa e não a exata prevalência de atividade física e suas associações na população de pessoas vivendo com HIV/aids de Ourinhos.

Tabela 3: Razões de prevalência ajustadas das variáveis dependentes (AFO, EFL, ALL e AFH) segundo variáveis sociodemográficas e de saúde em amostra de pessoas vivendo com HIV/aids no município de Ourinhos - SP, 2015-2016 ( $n=71)$.

\begin{tabular}{|c|c|c|c|c|}
\hline & AFO & EFL & ALL & AFH \\
\hline & $\begin{array}{l}\text { RP (IC95\%) } \\
\text { Ajustada }^{\text {a }}\end{array}$ & $\begin{array}{l}\text { RP (IC95\%) } \\
\text { Ajustada }^{\text {a }}\end{array}$ & $\begin{array}{l}\text { RP (IC95\%) } \\
\text { Ajustada }^{\text {a }}\end{array}$ & $\begin{array}{l}\text { RP (IC95\%) } \\
\text { Ajustada }^{a}\end{array}$ \\
\hline \multicolumn{5}{|l|}{ Sexo } \\
\hline Masculino & & $2,54(0,89-7,24)$ & & \\
\hline Feminino & & 1,00 & & \\
\hline \multicolumn{5}{|l|}{ Formação } \\
\hline Analfabeto/ EF1 & 1,00 & & & \\
\hline EF2/Médio/Superior & $1,24(0,39-3,92)$ & & & \\
\hline \multicolumn{5}{|l|}{ Classe Social } \\
\hline$A$ e $B$ & $3,32(1,34-8,25)^{*}$ & $2,86(1,11-7,36)^{*}$ & & \\
\hline$C, D$ e $E$ & 1,00 & 1,00 & & \\
\hline \multicolumn{5}{|l|}{ Tempo de tratamento } \\
\hline$<35$ (meses) & & & 1,00 & 1,00 \\
\hline Até 116 (meses) & & & $3,54(0,74-16,97)$ & $1,81(0,67-4,86)$ \\
\hline$>116$ (meses) & & & $3,83(0,79-18,50)$ & $2,00(0,75-5,35)$ \\
\hline \multicolumn{5}{|l|}{ Tempo de diagnóstico } \\
\hline$<50$ (meses) & & & 1,00 & 1,00 \\
\hline Até 150 (meses) & & & $2,98(0,62-14,47)$ & $2,47(0,88-6,89)$ \\
\hline$>150$ (meses) & & & $4,45(0,96-20,60)$ & $3,18(1,16-8,72)^{*}$ \\
\hline \multicolumn{5}{|c|}{ Pressão arterial diastólica $(\mathrm{mm} / \mathrm{Hg})$} \\
\hline$\leq 80$ & 1,00 & & 1,00 & 1,00 \\
\hline$>80$ & $2,01(0,87-4,65)$ & & $3,17(1,43-7,07)^{\star}$ & $1,81(0,93-3,52)$ \\
\hline
\end{tabular}

Legenda: AFO: Atividade física ocupacional; EFL: exercício físico no lazer; ALL: atividade física de lazer e locomoção; AFH: atividade física habitual; RP: razão de prevalência; IC95\%: intervalo de confiança de $95 \%$. aAnálise ajustada para sexo, faixa etária e formação; ${ }^{*} P<0,05$. 
Dessa forma conclui-se que pessoas vivendo com HIV/aids em tratamento na região de Ourinhos possuem baixos níveis de atividade física habitual, no entanto a maior parte da população investigada classifica sua saúde como boa. Além disso, verificou-se que a proporção de pessoas que praticam AFO, ALL e AFH foi maior respectivamente, entre aqueles de maior classe social, maior PAD e maior tempo de diagnóstico. Adicionalmente, também se observou que indivíduos de maior classe social e melhor saúde percebida apresentaram maior prevalência de EFL.

\section{AGRADECIMENTOS}

Agradecemos a Secretaria de Saúde e ao Serviço de Atendimento em Doenças Infecciosas de Ourinhos pela abertura do espaço para coleta de dados.

\section{REFERÊNCIAS}

1. AIDS Info. Glossary of HIV/AIDS-related terms. US; 2008. Disponível em: http://aidsinfo.nih.gov/ContentFiles/GlossaryHIVrelatedTerms. pdf. Acesso em: 30 abr. 2018

2. Brasil. Ministério da Saúde. Secretaria de Vigilância em Saúde. HIV-AIDS. Bol Epidemiol. 2016;5(1):6-8.

3. Gomes-Neto M, Conceição CS, Carvalho VO, Brites C. A systematic review of the effects of different types of therapeutic exercise on physiologic and functional measurements in patients with HIV/AIDS. Clinics. 2013;68(8):1157-67. http://dx.doi.org/10.6061/clinics/2013(08)16

4. Nosrat S, Whitworth JW, Ciccolo JT. Exercise and mental health of people living with HIV: A systematic review. Chronic IIIn. 2017;13(4):299-319. http://dx.doi.org/10.1177/1742395317694224

5. Mustafa T, Sy SF, Macera CA, Thompson SJ, Jackson KL, Selassie $A$, et al. Association between exercise and HIV disease progression in a cohort of homosexual men. Ann Epidemiol. 1999;9(2):127-31. http://dx.doi.org/10.1016/S1047-2797(98)00043-X

6. Mapstone M, Hilton TN, Yang H, Guido JJ, Luque AE, Hall WJ, et al. Poor aerobic fitness may contribute to cognitive decline in HIV-infected older adults. Aging Dis. 2014;4(6):311-9. http://dx.doi.org/10.14336/AD.2013.0400311

7. O'Brien K, Tynan AM, Nixon S, Glazier RH. Effectiveness of aerobic exercise for adults living with HIV: systematic review and meta-analysis using the Cochrane Collaboration protocol. BMC Infect Dis. 2016;16:182. http://dx.doi.org/10.1186/s12879-016-1478-2

8. Baecke JAH, Burema J, Frijters JER. A shorts questionnaire for the measurement of habitual physical activity in epidemiological studies. Am J Clin Nutr. 1982;36(5):936-42.

9. Florindo AA, Latorre MRDO, Santos ECM, Negrão CE, Azevedo LF, Segurado AAC. Validade e reprodutibilidade do questionário de Baecke para avaliação da atividade física habitual em pessoas vivendo com HIV/AIDS. Cad Saúde Pública. 2006;22(3):535-41. http://dx.doi.org/10.1590/S0102-311X2006000300008
10. Garber CE, Blissmer B, Deschenes MR, Franklin BA, Lamonte MJ, Lee IM, et al. American College of Sports Medicine position stand. Quantity and quality of exercise for developing and maintaining cardiorespiratory, musculoskeletal, and neuromotor fitness in apparently healthy adults: guidance for prescribing exercise. Med Sci Sports Exerc. 2011;43(7):1334-59.

http://dx.doi.org/10.1249/MSS.0b013e318213fefb

11. Associação Brasileira de Empresas de Pesquisa (ABEP). Critério de Classificação Econômica Brasil. Dados com base no levantamento socioeconômico. IBOPE; 2011.

12. Maliska ICA, Padilha MICS, Vieira M, Bastiani J. Percepções e significados do diagnóstico e convívio com o HIV/aids. Rev Gaúcha Enfermagem. 2009;30(1):85.

13. Ribeiro ARA, Guariglia DA, Pupulin ART, Teixeira DC, Greguol M, Cyrino ES. Barreiras pessoais para prática de atividade física percebidas por portadores de AIDS. Rev Educ Fis UEM. 2013;24(1):93-101.

http://dx.doi.org/10.4025/reveducfis.v24i1.16069

14. Paffenbarger Jr RS. Contributions of epidemiology to exercise science and cardiovascular health. Med Sci Sports Exerc. 1988;20(5):426-38. http://dx.doi.org/10.1249/00005768-198810000-00002

15. Colombrini MRC, Lopes MHBM, Figueiredo RM. Adesão à terapia antiretroviral para HIV/AIDS. Rev Esc Enferm USP. 2006;40(4):576-81.

http://dx.doi.org/10.1590/S0080-62342006000400018

16. Sávio KEO, Costa THM, Schmitz BAS, Silva EF. Sexo, renda e escolaridade associados ao nível de atividade física de trabalhadores. Rev Saúde Pública. 2008;42(3):457-63. http://dx.doi.org/10.1590/S0034-89102008005000018

17. Palma A. Atividade física, processo saúde-doença e condições sócio-econômicas: uma revisão da literatura. Revista Paulista de Educação Física. 2000;14(1):97-106.

http://dx.doi.org/10.11606/issn.2594-5904.rpef.2000.138022 